\title{
artigo
}

\section{Assistência de enfermagem as alteraçoes hemodinâmicas no período gravídico em pacientes com pré-eclâmpsia}

Nursing assistance to hemodynamic changes in the serious period in patients with pre-eclampsy

Asistencia de enfermería a los cambios hemodinámicos en el período grave en pacientes con pre-eclampsia

\begin{abstract}
RESUMO
Objetivo: Identificar os achados publicados na literatura científica sobre a assistência de enfermagem às alterações hemodinâmicas em mulheres com pré-eclâmpsia no ciclo gravídico. Método: trata-se de uma revisão integrativa da literatura, que reúne achados baseados em evidências científicas de estudos primários nas bases de dados Google Acadêmico, National Library of Medicine (PubMed), Biblioteca virtual de saúde (BVS), Scientific Eletronic Library Online (SciELO). Resultados: a amostra constituiu-se de cinco artigos primários publicados nos últimos cinco anos. Achados relacionados aos cuidados de enfermagem no que se refere a pré-eclâmpsia. Conclusão: através deste estudo foi possivel confirmar que a enfermagem tem papel importantíssimo no processo do cuidado às gestantes, proporcionando assistência, orientação e educação em saúde, evitando assim possíveis agravos.
\end{abstract}

DESCRITORES: Assistência de enfermagem; Pré-eclâmpsia; Hipertensão gestacional.

\section{ABSTRACT}

Objective: To identify the findings published in the scientific literature on nursing care for hemodynamic changes in women with pre-eclampsia in the pregnancy cycle. Method: this is an integrative literature review, which brings together findings based on scientific evidence from primary studies in the Google Scholar databases, National Library of Medicine (PubMed), Virtual Health Library (VHL), Scientific Eletronic Library Online ( SciELO). Results: the sample consisted of five primary articles published in the last five years. Findings related to nursing care with regard to pre-eclampsia. Conclusion: through this study it was possible to confirm that nursing has a very important role in the care process for pregnant women, providing assistance, guidance and health education, thus avoiding possible problems.

DESCRIPTORS: Nursing care; Pre eclampsia; Gestational hypertension.

\section{RESUMEN}

Objetivo: Identificar los hallazgos publicados en la literatura científica sobre el cuidado de enfermería de los cambios hemodinámicos en mujeres con preeclampsia en el ciclo del embarazo. Método: es una revisión integradora de la literatura, que reúne hallazgos basados en evidencia científica de estudios primarios en Google Scholar, National Library of Medicine (PubMed), Virtual Health Library (VHL), Scientific Electronic Library Online ( SciELO). Resultados: la muestra estuvo conformada por cinco artículos primarios publicados en los últimos cinco años. Hallazgos relacionados con los cuidados de enfermería en relación con la preeclampsia. Conclusión: a través de este estudio se pudo constatar que la enfermería tiene un papel muy importante en el proceso de atención a la gestante, brindando asistencia, orientación y educación para la salud, evitando así posibles problemas. DESCRIPTORES: Atención de enfermería; Preeclampsia; Hipertensión gestacional.

RECEBIDO EM: 18/01/2021 APROVADO EM: 01/02/2021

\section{Michelle de Carvalho Veloso Café}

Graduanda em enfermagem pela Faculdade Adventista da Bahia BA-Brasil.

ORCID: 0000-0002-2329-6356

\section{Maressa Farias Santana}

Graduanda em enfermagem pela Faculdade Adventista da Bahia BA-Brasil. Graduanda em Farmácia pela Faculdade Metropolitana de Maringá.

ORCID: 0000-0001-9751-8866 


\section{Gabriel Lucas Pereira Guimarães}

Tecnólogo em Secretariado, Pós-graduando em Administração e Auditoria em Serviços de Saúde pela Faculdade Dom Alberto, Graduando em Enfermagem pela Faculdade Adventista da Bahia. BA-Brasil.

ORCID: 0000-0002-4669-6715

\section{Anne Eugênia Ledo Gonçalves Viana}

Graduanda em enfermagem pela faculdade adventista da Bahia. BA-Brasil.

ORCID: 0000-0002-6607-354X

\section{Rosangela Jeniffer Soares Rosa}

Graduanda em enfermagem pela Faculdade Adventista da Bahia. BA-Brasil. ORCID: 0000-0002-3380-5601

\section{Paula Paulina Costa Tavares}

Enfermeira. Mestrado Profissional em Promoção da saúde pelo Centro universitário Adventista de São Paulo. Docente do curso de Enfermagem da Faculdade Adventista da Bahia. BA-Brasil.

ORCID: 0000-0002-9275-8884

\section{INTRODUÇÃO}

D e acordo com a Organização Mundial da Saúde (OMS), quase 10\% das gestantes em todo o mundo são acometidas pelos distúrbios hipertensivos da gravidez, os quais ocupam o segundo lugar no ranking das causas de morte materna, ficando atrás apenas das hemorragias. Este grupo de doenças e condições inclui pré-eclâmpsia ( $\mathrm{PE}$ ), eclâmpsia, hipertensão gestacional e hipertensão crônica ${ }^{(1)}$. Dentre os distúrbios hipertensivos, a PE configura-se como o principal acometimento às gestantes, tornando-se uma das maiores causas de mortalidade e morbidade relacionada à gravidez no mundo, afetando cerca de 5-8\% de todas as gestações ${ }^{(2)}$.

A PE caracteriza-se pelo surgimento de hipertensão após a $20^{\mathrm{a}}$ semana de gestação (ou antes, em caso de doença trofoblástica gestacional ou hidropsia fetal), estando comumente acompanhada de proteinúria, tendendo a desaparecer até 12 semanas após o parto. Quando não há presença de proteinúria, o diagnóstico é identificado pela elevação da pressão arterial (PA), acompanhada de cefaléia, distúrbios visuais, dores abdominais, plaquetopenia e aumento de enzimas hepáticas ${ }^{(2)}$.

A conduta que visa a prevenção das síndromes hipertensivas da gestação inclui a identificação precoce dos fatores de risco com monitorização, intervenção e controles destes, e aferição da pressão arterial (PA) em todas as consultas de pré-natal e controle rigo- roso em pacientes que já tem histórico pessoal de hipertensão crônica ${ }^{(3)}$.

Destacam-se como principais agravantes para o surgimento da PE, a primigestação, histórico familiar e pessoal de doenças cardiovasculares como hipertensão e diabetes. Por esse motivo, pacientes com esses fatores de risco devem receber atenção especial durante as consultas de pré-natal para que o diagnóstico seja realizado precocemente, visto que a implementação de medidas preventivas no período gravídico-puerperal garante melhor prognóstico e assistência materno-fetal ${ }^{(4)}$.

Analisando este contexto e tendo em vista a inexistência de um tratamento curativo, a pré-eclâmpsia exige detecção antecipada, manejo clínico adequado e monitorização eficaz. Assim, a assistência de enfermagem durante o ciclo gravídico-puerperal desempenha importante papel no controle dos agravos da pré-eclâmpsia, pois o diagnóstico precoce e a assistência qualificada são fatores indispensáveis para um bom prognóstico ${ }^{(5)}$.

Considerando a relevância da temática, a pergunta norteadora deste estudo é: Quais as evidências científicas disponíveis na literatura sobre a assistência de enfermagem às alterações hemodinâmicas no período gravídico em pacientes com pré-eclâmpsia? O objetivo proposto para esta revisão é identificar e descrever achados do que se tem publicado na literatura científica sobre a avaliação e assistência de enfermagem à alteração hemodinâmica em mulheres com pré-eclâmpsia no ciclo gravídico.

\section{MÉTODO}

Trata-se de uma revisão integrativa da literatura, que reúne achados baseados em evidências científicas de estudos primários, cuja temática envolve artigos referentes à assistência de enfermagem às alterações hemodinâmicas no ciclo gravídico em pacientes com pré-eclâmpsia.

A revisão integrativa tem por finalidade realizar uma síntese de resultados em pesquisas analisadas à luz da literatura científica sobre um determinado assunto ou questão. Tendo potencial para contribuir no desenvolvimento do conhecimento e nas práticas assistenciais em enfermagem ${ }^{(6)}$.

Esta revisão seguiu as seguintes etapas: 1) Elaboração do problema da pesquisa. 2) Busca nas bases de dados com aplicação dos critérios de inclusão e exclusão; 3) Leitura e análise crítica dos artigos; 4) Categorização com vistas a organizar os achados; 5) Interpretação e discussão dos resultados e 6) concluído mediante apresentação dos resultados e análise crítica dos dados obtidos pertinentes à pesquisa de forma descritiva.

O levantamento dos artigos foi realizado em agosto e setembro de 2020, utilizando as seguintes plataformas como bases de dados: Google Acadêmico, National Library of Medicine (PubMed), Biblioteca virtual de saúde (BVS), Scientific Eletronic Library Online (SciELO). As palavras chaves foram estabelecidas de acordo com os Descritores em Ciências 


\section{artigo}

Café, M.C.V.; Santana, M.F.; Guimarães, G.L.P.; Viana, A.E.L.G.; Rosa, R.J.S.; Tavares, P.P.C.

Assistência de enfermagem as alteraçoes hemodinâmicas no período gravídico em pacientes com pré-eclâmpsia

da Saúde (DeCS), sendo: assistência de enfermagem, pré-eclâmpsia, hipertensão gestacional. Como critério de inclusão, foram considerados os artigos originais, completos, com livre acesso e publicados em português e inglês, entre os anos 2015 a 2020, disponíveis nas bases de dados previamente citadas. Foram adotados como critérios de exclusão artigos que apresen- tavam fuga do tema, dissertações, teses, e monografias, resumos ou com acesso pago.

Foram lidos um total de 411 títulos de artigos e selecionados aqueles que atendiam o tema. Em seguida foi realizada a leitura dos resumos, sendo selecionados 36 artigos que atenderam aos critérios de inclusão. Por fim, após a leitura completa de 13 artigos, 05 foram selecionados.

Figura 1. Fluxograma das informações da pesquisa nas bases: Google Acadêmico, National Library of Medicine (PubMed), Biblioteca virtual de saúde (BVS), Scientific Eletronic Library Online (SciElo). Mapeando o número de registros identificados, incluídos e excluídos, Cachoeira, BA, Brasil, 2020.

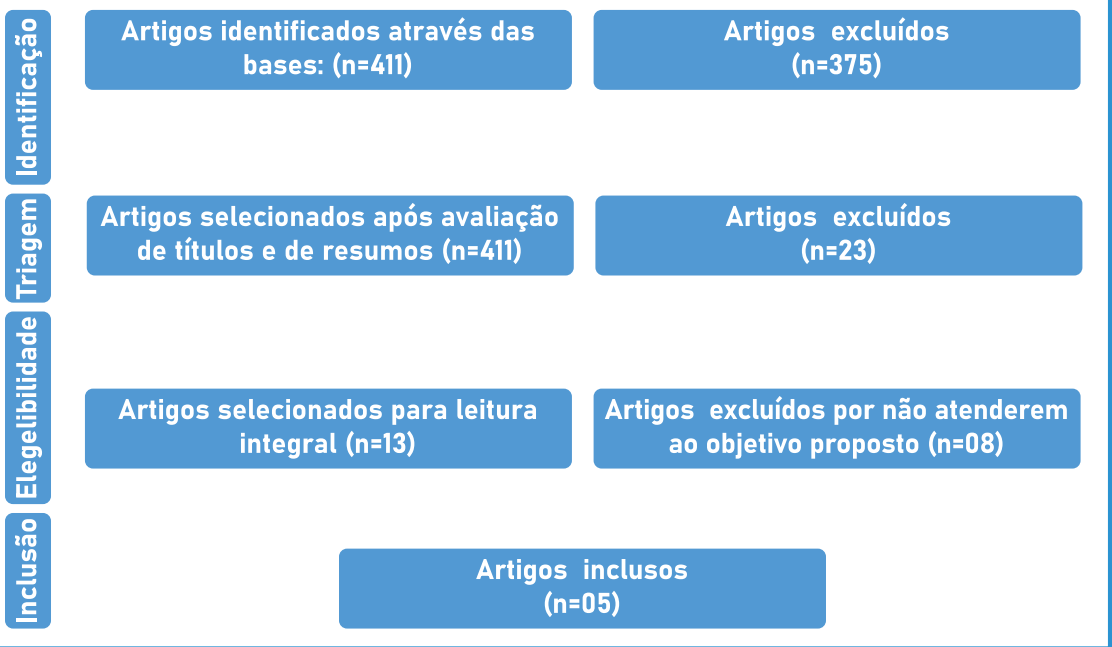

\section{RESULTADOS}

A amarração teórica no Quadro 1 refere-se aos autores, temática dos artigos, resultados e revista publicada e ano de publicação. Dos cinco estudos selecionados, dois (02) artigos foram encontrados na base de dados do Google Acadêmico, outros três (03) artigos foram da BVS e apenas um (01) na SciELO. Foi realizada a síntese crítica dos resultados, no que se refere à assistência de enfermagem às alterações hemodinâmicas no período gravídico em pacientes com pré-eclâmpsia.

\section{DISCUSSÃO}

A assistência de enfermagem às gestantes com síndrome hipertensiva é primordial para um bom prognóstico, requer do profissional enfermeiro uma associação entre autonomia, senso crítico e conhecimento técnico-científico, justaposto à assistência de uma equipe multiprofissional capacitada, tornando o processo de trabalho dinâmico e resolutivo ${ }^{(6)}$. Nesse contexto, a enfermagem dentro da equipe multidisciplinar desenvolve papel colaborativo direto, proporcionando otimização dos cuidados às pacientes ${ }^{(9)}$.

Quadro 1. Artigos selecionados nas bases de dados entre os anos de 2015 a 2020

\begin{tabular}{|c|c|c|c|c|}
\hline $\mathbf{N}^{\circ}$ & AUTORES & TÍTULO & RESULTADOS & REVISTA \\
\hline 1 & $\begin{array}{l}\text { Santana RS, Costa } \\
\text { ACRR, Fontes FLL, } \\
\text { Carvalho FR, Moura } \\
\text { FF, Duarte JM, et al }{ }^{(7)}\end{array}$ & $\begin{array}{l}\text { Importância do conheci- } \\
\text { mento sobre sinais e sin- } \\
\text { tomas da pré-eclâmpsia } \\
\text { para implementação dos } \\
\text { cuidados de Enfermagem }\end{array}$ & $\begin{array}{c}\text { O cumprimento da prescrição medicamentosa e } \\
\text { aferição dos sinais vitais, em especial pressão arte- } \\
\text { rial, foram os cuidados comumente implementados. } \\
\text { Também verificou-se que nenhuma das entrevis- } \\
\text { tadas mencionou cuidados com a vitalidade fetal, } \\
\text { priorizando-se apenas a monitorização da mãe; o } \\
\text { que evidencia fragilidade da assistência realizada no } \\
\text { quesito binômio mãe-filho }\end{array}$ & $\begin{array}{l}\text { Revista Eletrônica } \\
\text { Acervo Saúde } 2019\end{array}$ \\
\hline 2 & $\begin{array}{c}\text { Oliveira GS, Paixão } \\
\text { GPN, Fraga CDS, } \\
\text { Santos MKR, Santos } \\
\text { MA. }{ }^{(8)}\end{array}$ & $\begin{array}{c}\text { Assistência de } \\
\text { enfermeiros na síndrome } \\
\text { hipertensiva gestacional } \\
\text { em hospital de baixo risco } \\
\text { obstétrico. }\end{array}$ & $\begin{array}{c}\text { Foram consolidados em três categorias, a saber: } \\
\text { abordagem do enfermeiro às mulheres com } \\
\text { síndrome hipertensiva gravídica; fatores que } \\
\text { dificultam uma adequada assistência; atuação } \\
\text { essencial do enfermeiro para preservação da vida } \\
\text { do binômio mãe-filho. }\end{array}$ & $\begin{array}{c}\text { Revista CUIDARTE. } \\
2017 .\end{array}$ \\
\hline 3 & $\begin{array}{l}\text { Silva CML, Miguel } \\
\text { TBAR, Borges JAM, } \\
\text { Aguiar AC, Reis PAM. }{ }^{(3)}\end{array}$ & $\begin{array}{l}\text { Assistência de enfer- } \\
\text { magem à gestante com } \\
\text { Síndrome neurológica: pré- } \\
\text {-eclâmpsia e eclampsia }\end{array}$ & $\begin{array}{l}\text { Após análise houve três categorias temáticas: assis- } \\
\text { tência prestada a paciente com pré-eclâmpsia; per- } \\
\text { cepção da equipe de enfermagem sobre a Sistema- } \\
\text { tização da Assistência de enfermagem. Sentimentos } \\
\text { das pacientes durante a assistência de enfermagem. }\end{array}$ & $\begin{array}{l}\text { Revista Perspectiva } \\
\text { da Saúde } 2019\end{array}$ \\
\hline
\end{tabular}




\begin{tabular}{|l|c|c|c|c|}
\hline 4 & $\begin{array}{c}\text { Oliveira KKPA, An- } \\
\text { drade SSC, Silva FMC, } \\
\text { Meneses LBA, Leite } \\
\text { KNS, Oliveira SHS. }{ }^{(9)}\end{array}$ & $\begin{array}{c}\text { Assistência de enfer- } \\
\text { magem a parturientes } \\
\text { acometidas por pré-e- } \\
\text { clâmpsia. }\end{array}$ & $\begin{array}{c}\text { As ideias centrais foram: Cuidado voltado a pré-e- } \\
\text { clampsia; Sentimentos quanto à hospitalização; } \\
\text { Orientações dietéticas e de controle da pressão } \\
\text { arterial; e Atendimento das necessidades individuais } \\
\text { e avaliação dos resultados. }\end{array}$ & $\begin{array}{c}\text { Revista de Enferma- } \\
\text { gem UFPE On line } \\
2016 .\end{array}$ \\
\hline 5 & Phillips C, Boyd M. (10) & $\begin{array}{c}\text { Assessment, Manage- } \\
\text { ment, and Health Impli- } \\
\text { cations of Early-Onset } \\
\text { Pré-eclampsia. }\end{array}$ & $\begin{array}{c}\text { A prioridade nos cuidados de enfermagem é a ava- } \\
\text { liação contínua vigilante para sinais de agravamento } \\
\text { da doença, considerando o caráter progressivo de } \\
\text { pré-eclâmpsia. O objetivo é manter uma mulher e seu } \\
\text { feto / recém-nascido seguro antes, durante e após o } \\
\text { nascimento. Mulheres com a pré-eclâmpsia pode ser } \\
\text { instável por vários motivos. }\end{array}$ & $\begin{array}{c}\text { Nursing for Women's } \\
\text { Health } 2016\end{array}$ \\
\hline Fonte: Café MCV, Santana MF, Guimarães GLP, etal. 2021 & & & \\
\hline
\end{tabular}

$\mathrm{O}$ acesso ao pré-natal ainda no primeiro trimestre gestacional tem sido considerado indicativo de qualidade assistencial das Unidades de Atenção Básica. Os cuidados do pré-natal são imprescindíveis para o diagnóstico antecipado de alterações e consequentemente realização de intervenções referentes à vulnerabilidade da saúde da gestante e da criança. É fundamental o acompanhamento e a abordagem multidisciplinar bem como a garantia de uma assistência acolhedora, singular e integral. Além disso, os profissionais de saúde devem investir em ações que possibilitem a educação e cuidado em saúde, objetivando o bem estar tanto da gestante quanto do feto. Apesar disso, alguns fatores dificultam a assistência de enfermagem às pacientes, principalmente no contexto da atenção básica, como falhas no acompanhamento pré-natal, ausência de informações sobre possíveis alterações e questionamentos referentes a sinais e sintomas que levem a investigação de possíveis patologias ${ }^{(6,11)}$.

Em virtude disso, a atuação da equipe multidisciplinar da atenção básica é imprescindível, uma vez que as pacientes quando não são bem orientadas sobre o momento do parto, acabam desenvolvendo quadro de medo e ansiedade, podendo colaborar para um desequilíbrio ainda maior do quadro clínico da mulher no momento do parto. O despreparo para o diagnóstico de uma gravidez de risco iminente, pressupõe a ausência de educação continuada e/ou deficiência na assistência prestada. A partir dessa constatação, percebe-se a importância da educação em saúde para a garantia de uma assistência qualificada ${ }^{(7)}$.

$\mathrm{O}$ processo de assistência passa por constantes mudanças e nesse sentido uma busca por atualização faz-se necessário. A educação continuada é um caminho viável de aprimoramento profissional e assistencial, fazendo assim com que a equipe de enfermagem tenha acesso a um conjunto de experiências que estão diretamente ligadas à formação inicial possibilitando que o profissional mantenha, aumente ou melhore significativamente suas competências assistenciais, culminando assim em ações de saúde de qualidade, resolutivas, humanizadas e em tempo adequado ${ }^{(7,9)}$.

A educação continuada para os trabalhadores da área da saúde requer empenho para o aperfeiçoamento de condutas que promovam o desenvolvimento do processo de trabalho. Para que o método atinja sua eficácia é necessário que sejam desenvolvidas estratégias de educação que impulsionem a participação dos trabalhadores e dessa forma capacite os profissionais no exercício de suas atividades laborativas. Por essa razão, ao encorajar o profissional à mudança, as ações educativas tornam-se capaz de minimizar as dificuldades nas práticas de ensino, e por esse motivo objetiva uma enfermagem apta a traçar metas a serem alcançadas por toda equipe ${ }^{(12)}$. Desta forma, este conjunto de ações possibilita ao profissional enfermeiro atualizações constantes, que podem, inclusive, viabilizar durante o pré-natal, o diagnóstico precoce dos distúrbios hipertensivos da gestação, da qual a pré-eclâmpsia faz parte.

Dentre as possibilidades de apresentação da pré-eclâmpsia está a de início precoce, antes das 34 semanas de gestação, a qual predispõe possíveis eventos adversos como parto prematuro. Os casos de PE que são detectados antecipadamente representam apenas um pequeno número quando comparados à quantidade total de casos da doença, isto significa que as mulheres que recebem o diagnóstico tardiamente, carecem de triagem específica e apurada para detecção de alterações clínicas ${ }^{(10)}$.

Embora o diagnóstico da PE de início precoce seja desafiador, visto que a progressão da doença ocorre de forma insidiosa por semanas até a manifestação dos primeiros sintomas, que possibilitem a autopercepção por parte da mulher, para que a mesma os relate no momento da consulta, faz-se necessário devida orientação para oportuna identificação e manejo clínico adequado, uma vez que a PE é mais que um simples distúrbio hipertensivo e demanda cuidados específicos ${ }^{(10)}$.

O desconhecimento sobre a doença pode ser sanado mediante assistência pré-natal efetiva, com acompanhamento contínuo, tendo uma assistência durante a gestação pautada em orientações e resposta às dúvidas. Existem ainda, outros fatores que interferem na qualidade da assistência, como a falta de avaliação fetal, deficiência de conhecimento para manusear os equipamentos e até mesmo desconhecimento sobre a própria doença ${ }^{(7)}$.

Dessa forma, a avaliação do bem estar fetal e crescimento geral, juntamente com o monitoramento e aferição da pressão arterial da gestante, são etapas imprescindíveis para o encaminhamento correto de pacientes com pré-eclâmpsia. Para além disso, o acolhimento através da escuta qualificada também desempenha grande importância durante a assistência de enfermagem, visto que demonstra 


\section{artigo}

preocupação com a gestante tendo como base as singularidades da mesma, e por esse motivo o momento da admissão é imprescindível para o fortalecimento do vínculo entre o profissional e a mãe. Em razão disso, personalizar o atendimento é extremamente importante, uma vez que a assistência individual proporciona maior conhecimento sobre o quadro clínico de cada paciente ${ }^{(6)}$.

No ambiente hospitalar o enfermeiro precisa estar capacitado para identificar as manifestações clínicas e realizar os primeiros atendimentos de modo assertivo, estabelecendo condutas como: aferição da pressão arterial quatro vezes ao dia, garantia de repouso no leito, avaliação de proteinúria, orientaçôes para verificação materna diária dos movimentos fetais e observação dos sinais e sintomas das síndromes ${ }^{(7)}$.

Destaca-se ainda como cuidado de enfermagem comumente implementado, o cumprimento da prescrição medicamentosa. Conforme a Federação Brasileira das Associações de Ginecologia e Obstetrícia (FEBRASGO), a droga de primeira escolha utilizada em casos de crise hipertensiva é a nifedipina via oral (VO), porém a hidralazina intravenosa (IV) ou intramuscular (IM) também pode ser utilizada como alternativa ao tratamento ${ }^{(6,11)}$.

Em relação aos cuidados com a vitalidade fetal, muitas vezes se prioriza apenas a monitorização da gestante; evidenciando um déficit na conduta de enfermagem prestada ao binômio gestante-feto. É importante salientar que quando a gestante se encontra hospitalizada, o feto deve ser auscultado todos os dias, e a movimentação fetal também deve ser observada. Em casos de pré-eclâmpsia leve, o bem estar fetal deve ser avaliado uma vez por semana ou conforme alteração do estado materno. É importante sinalizar também que no momento do diagnóstico da PE, deve ser solicitada uma ecografia para monitorar o desenvolvimento fetal ${ }^{(6,9)}$.

\section{CONCLUSÃO}

Através deste estudo foi possível confirmar que a enfermagem tem papel importantíssimo no processo do cuidado às gestantes, proporcionando assistência, orientação e educação em saúde e evitando assim possíveis agravos. Nessa perspectiva os cuidados de enfermagem achados neste estudo envolvem: aferição da pressão arterial, detecção precoce, escuta qualificada, orientações adequadas e em tempo oportuno às gestantes sobre os sinais e sintomas, cumprimento da prescrição medicamentosa, cuidados com a vitalidade fetal e necessidade de educação continuada para o aperfeiçoamento da assistência prestada. E

\section{REFERÊNCIAS}

1. Organização Mundial da Saúde. Recomendações da OMS para prevenção e tratamento da pré-eclâmpsia e da eclâmpsia: Antecedentes [Internet]. Genebra, Suíça; 2016 [cited 2020 Aug 2]. Available from: https://apps.who.int/iris/bitstream/handle/10665/44703/9789248548338_por.pdf?sequence=11

2. Ministerio da Saúde. Gestação de Alto Risco Manual Técnico [Internet]. Brasilia -DF; 2010 [cited 2020 Aug 3]. Available from: https:// bvsms.saude.gov.br/bvs/publicacoes/gestacao_alto_risco.pdf

3. Silva CML, Miguel TBAR, Borges JAM, Aguiar AC, Reis PAM. Assistência de enfermagem à gestante com síndrome neurológica: Pré eclâmpsia e eclâmpsia. Revista Perspectiva da Saúde [Internet]. 2019 [cited 2020 Sep 20];1(1):39-48. Available from: http://unigrande. edu.br/wp-content/uploads/2020/01/Perspectiva_da_Saude.pdftpage $=40$

4. Aguiar LRS, Silva MGP, Feitosa WF, Cunha KJB. Análise de estudos sobre as condutas de enfermagem no cuidado à gestante com doença hipertensiva. Revista interdisciplinar [Internet]. 2014 [cited 2020 Aug 3];7(1):204-215. Available from: https://revistainterdisciplinar.uninovafapi.edu.br/index.php/revinter/article/view/252/ pdf_111

5. Oliveira GS, Paixão GPN, Fraga CDS, Santos MKR, Santos MA. Assistência de enfermeiros na síndrome hipertensivo gestacional em hospital de baixo risco obstétrico. Revista Cuidarte [Internet]. 2017 [cited 2020 Aug 3];8 Available from: http://www.scielo.org.co/scielo. php?pid=S2216-09732017000201561\&script=sci_abstract\&tl$\mathrm{ng}=\mathrm{pt}$

6. Mendes KDS, Silveira RCCP, Galvão CM. Revisão integrativa: método de pesquisa para a incorporação de evidências na saúde e na enfermagem. Texto Contexto Enferm. 2008;17(4):758-764.
7. Santana RS, Costa ACRR, Fontes FLL, Carvalho FR, Moura FF, Duarte JM, et al. Importância do conhecimento sobre sinais e sintomas da pré-eclâmpsia para implementação dos cuidados de Enfermagem. Revista Eletrônica Acervo Saúde [Internet]. 2019 Sep 01 [cited 2020 Sep 2]; Available from: https://acervomais.com.br/index.php/saude/ article/view/1425

8. Oliveira GS, Paixão GPN, Fraga CDS, Santos MKR, Santos MA. Assistência de enfermeiros na síndrome hipertensiva gestacional em hospital de baixo risco obstétrico. Portal de Revistas de Enfermagem [Internet]. 2017 Aug 08 [cited 2020 Sep 2]; Available from: https:// pesquisa.bvsalud.org/portal/resource/pt/biblio-904769

9. Oliveira KKPA, Andrade SSC, Silva FMC, Meneses LBA, Leite KNS, Oliveira SHS. Assistência de enfermagem às parturientes afetadas pela pré eclâmpsia [Internet]. Recife: Journal of Nursing; 2016 MAIO [cited 2020 Sep 9]. Available from: https://periodicos.ufpe.br/revistas/ revistaenfermagem/article/download/13556/16335

10. Phillips C, Boyd M. Assessment, Management, and Health Implications of Early-Onset Preeclampsia. Nursing for Women's Health. 2016; 20(4)

11. Federação Brasileira das Associações de Ginecologia e Obstetrícia (FEBRASGO). Pré-eclâmpsia nos seus diversos aspectos [Internet]. São Paulo: Federação Brasileira das Associações de Ginecologia e Obstetrícia (FEBRASGO); 2017 [cited 2020 Sep 9]. Available from: https://www.febrasgo.org.br/media/k2/attachments/12-PRE_ ECLAyMPSIA.pdf

12. Peixoto LS, Gonçalves LC, Costa TD, Tavares CMM, Cavalcanti ACD, Cortez EA. Educação permanente, continuada e em serviço: desvendando seus conceitos. Enfermería global: Revista electrónica trimestral de Enfermería. 2013;(29):324-340 